\title{
The Recent Trends in Fibre Optic Communication
}

\author{
Eng. ISSA OBAID ALORF \\ Electricity \& Electronics Department Higher Institute of Communication \& Navigation The Public Authority \\ For Applied Education \& Training
}

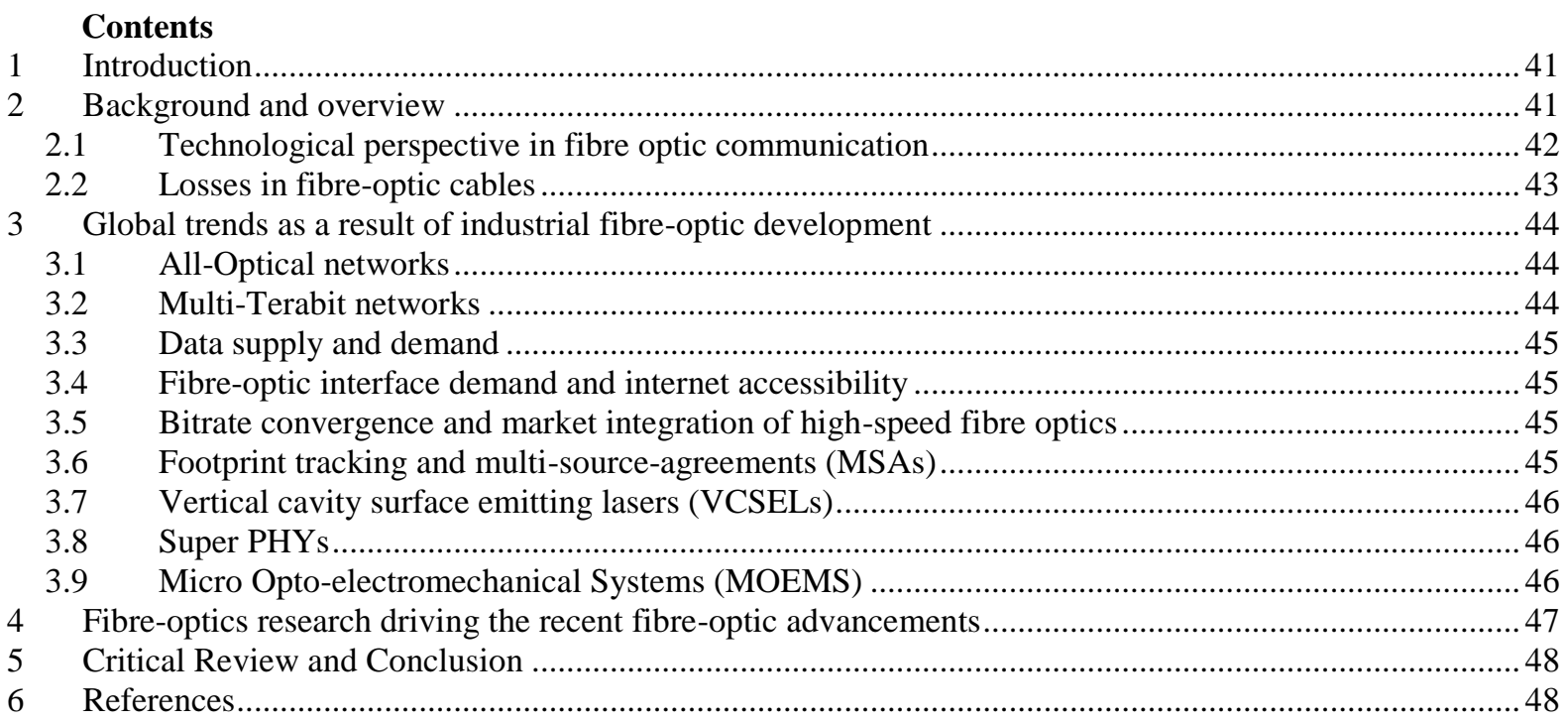

\section{List of Figures}

Figure 1: The global bandwidth demand as adapted from the ITU data (Routray 2014) 42

Figure 2: Two unique indexing types describing geometrical optics properties of fibre optic cables (Agrawal,

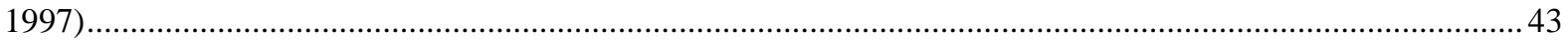

Figure 3: DWDM-based multi-terabit networks (Fibreopticshare, 2013) ………………................................ 44 Figure 4: A fire-optic micro-machined beam splitter based on the polarisation principle (Dragoman and

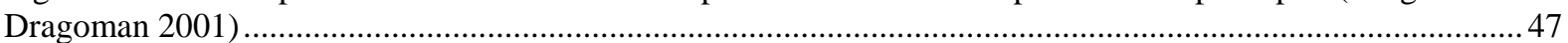

Figure 5: Block diagram of a 40Gbps DP-QPSK coherent transceiver as adapter (Laperle et al. 2008) ..............47

Figure 6: A commercial 40 Gbps DP-QPSK coherent transceiver (Roberts et al. 2009) ..................................... 48

\section{Introduction}

Rudimentary work on waveguides for long-range communication originally started in 1964 for wideband signals. Initially, the advancements in this domain suffered from significant challenges including dielectric losses, attenuation, inter/intra-model dispersion and micro/macro band losses. It was not until 1980s that optical communication emerged as a feasible mean of communication. However, the medium still suffered from major drawbacks. For instance, there were no optical amplifiers at that time. Moreover, the nodes/repeaters had re-amplification, retiming and reshaping (3R) regeneration processes that were done in the electrical domain which is commonly termed as the optical-electrical-optical (O-E-O) conversions. This resulted in a transformation from optical to electrical and back to optical domain which had its own performance bottlenecks. Despite these shortcomings, optical communication has seen increasing modifications and innovations that are improving speed, the core or the access, and the optical burst switching mechanisms (Pedrola et al. 2011).

Based on the rapidly improving and advancing backdrop of fibre optic communication, this document presents a comprehensive review of the recent trends in fibre optic communication. In order to assess and critically analyse these trends, Section 2 presents a background review of the fibre optic communication architecture, various loss types, and various other ongoing improvements. Section 3 presents an in-depth analysis of the global industrial trends in the fibre optic industry. Section 4 presents the relevant fibre optic research and finally concludes with a critical analysis in Section 5.

\section{Background and overview}

The underlying phenomenon of fibre optic communication is based upon total internal reflection which guides light trajectory to facilitate signal/data transfer. The idea was originally introduced in 1854 , though the 
core transfer medium, the glass fibres were made in 1920s. However, the actual fibre-optic communication only became possible in 1950s when "cladding layers" were introduced to improve the signal guidance characteristics of clad fibre optic cables. Prior to 1970s, the optical fibre technology was primarily used in short distance medical imaging and was not deemed suitable for communication purposes due to high losses in the range of $1000 \mathrm{~dB} / \mathrm{km}$. The situation improved in 1966s when Charles K Kao of IEE revealed fewer than $20 \mathrm{~dB} / \mathrm{km}$ losses. The first telephone service via optical was developed by AT\&T in 1977 and the first transatlantic telecommunication (TAT) fibre optic communication cable TAT-8 was deployed. The erbium doped fibre amplifier (EDFA) was developed in the University of Southampton and TAT-8 started service at $1.3 \mu \mathrm{m}$ (Routray 2014). By 1998, data traffic volume via fibre-optic cables outstripped voice traffic due to phone calls due to an exponential increase in the internet services. Since that time, internet traffic has increased from a $45 \%$ growth rate to a $100 \%$ growth rate per year. The international bandwidth demand during the past decade has since increased from 2 to 80 TBps (See Figure 1).

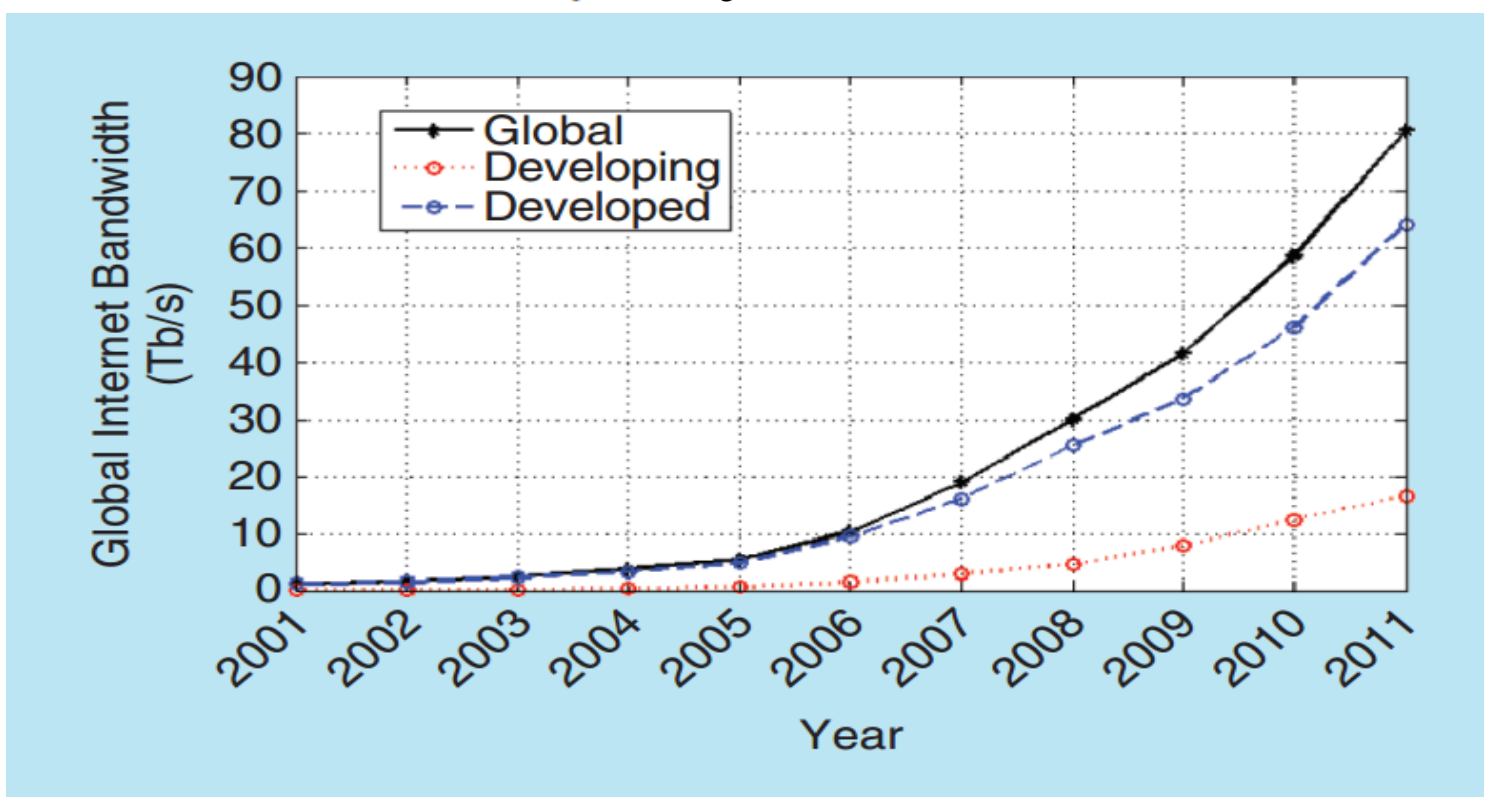

Figure 1: The global bandwidth demand as adapted from the ITU data (Routray 2014)

The current fibre optic trends focus on a number of core factors including transparency and coherence. The O-E$\mathrm{O}$ conversion in the intermediate repeaters and nodes is regarded as the absence of transparency. Transparency is thus an all-optical communication without any change from optical to electrical and then optical again. According to Routray (2014), the first level of transparency is opaque where the reamplification and reshaping is done at all repeaters. The second type is translucent where the $3 \mathrm{R}$ processing is performed at a limited number of repeaters whereas at the remaining repeaters it may just be at $1 \mathrm{R}$ or $2 \mathrm{R}$. In the third type, the processing at all nodes may just be limited to $1 \mathrm{R}$ or $2 \mathrm{R}$.

Coherent detection is popular in wireless networks where improved data transfer rate and better quality are offered to optical systems (Charlet 2008). The optical modulation format has become a popular area of research to feed the coherent receivers. The coherent detectors are capable of handling data traffic in the multiples of Tbps and also facilitate schemes such as orthogonal frequency division multiplexing (OFDM). The OFDM provides useful traits such as spectral efficiency, improved signal quality, and cost minimisation.

\subsection{Technological perspective in fibre optic communication}

In its most basic form, a fibre optic cable consists of an inner cylindrical core covered by a refractive cladding. The refractive index of this cladding is less than the inner core. There are two unique types of such refractive cores with abruptly changing index cores called the "step-index" fibres and the gradually decreasing index fibres termed the "graded-index" fibres (Makouei 2013). 


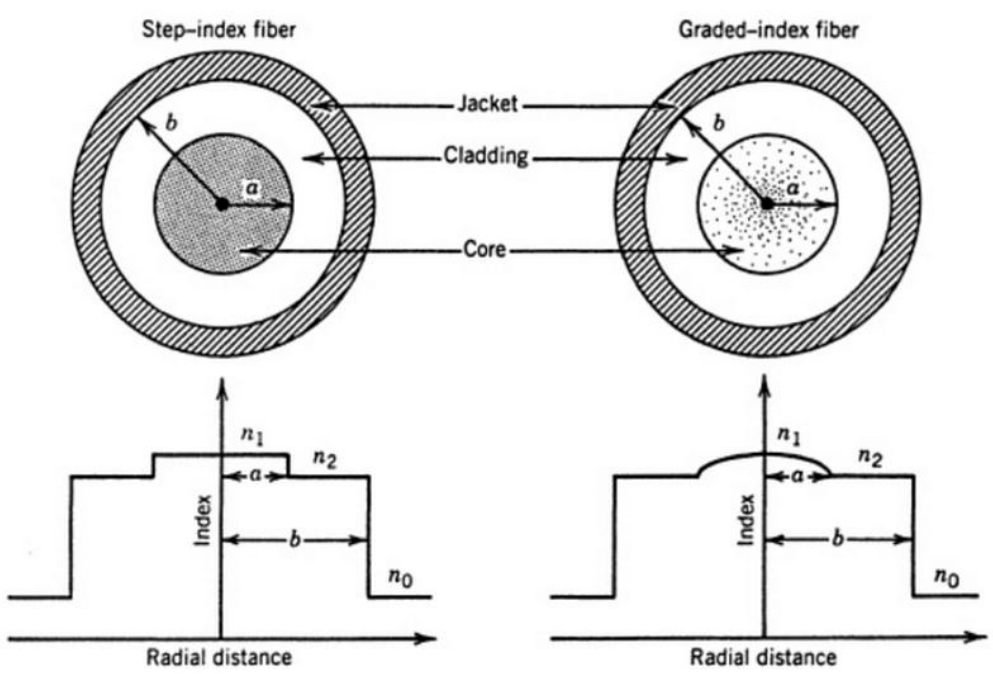

Figure 2: Two unique indexing types describing geometrical optics properties of fibre optic cables (Agrawal, 1997)

\subsection{Losses in fibre-optic cables}

Losses in fibre-optic cables generally occur due to absorption, scattering, dispersion and bending of cables. There are two types of bending losses - macroscopic and microscopic (Cheng and Tsao 2005). The former, macroscopic type occurs when bends in the cable assembly cause certain modes not to be reflected thereby resulting in losses due to cladding. In the latter, microscopic bending type, slight bends in the cable surface causes light to be reflected a certain angles when there is no more reflection.

Absorption losses occur due to heating of ionic impurities resulting in light diffusion at the end of the cable. There are two types of absorption losses - intrinsic and extrinsic losses. The intrinsic absorption occurs by the interaction with one or more glass components (Xu et al. 2001). The phenomenon occurs when photonic units interact with electrons in the valence band. The process results in excitation to a higher energy medium closer to the ultraviolet region. Extrinsic absorption is also termed impurity absorption which results from the presence of transitory metal ions (e.g. iron, chromium or cobalt) from $\mathrm{OH}$ ions.

Dispersion losses occur when optical signal travelling within a cable is distorted. The distortion is either intermodal or intramodal (Yaman et al. 2006). Intermodal distortion occurs as a result of pulse broadening due to propagation delay differences between modes in a multi-mode fibre. In the intramodal dispersion, pulse spreading occurs within a single mode due to material or waveguide dispersion. In material dispersion which is also regarded as spectral or chromatic dispersion, results due to refractive index variation. The variation occurs as a function of wavelength which causes pulse spreading even when various wavelengths follow the same trajectories. The waveguide dispersion occurs when the optical signal is passed through the fibre. During this process, $80 \%$ of the optical signal strength is confined to the core whereas $20 \%$ is confined into the cladding (See Figure 2 for core and cladding).

Scattering losses occur as a result of microscopic variations in the material density. The losses also occur due to compositional fluctuations, structural inhomogeneities and assembly/manufacturing faults (Lines 1984).

Linear scattering generally present three types of scattering losses:

- Rayleigh scattering losses

- Mie scattering losses

- Waveguide scattering losses

Nonlinear scattering losses are generally of two types:

- $\quad$ Stimulated Brillouin Scattering (SBS)

- $\quad$ Stimulated Raman Scattering (SRS)

Rayleigh scattering losses occur due to microscopic variation in the fibre material where irregular molecular or atomic density distribution results in Rayleigh scattering losses. This happen due to the presence of various acid compositions in the optical glass such as $\mathrm{SiO}_{2}$ or $\mathrm{P}_{2} \mathrm{O}_{5}$. Moreover, compositions and fluctuations can take place due to the presence of many oxides that lead of Raleigh scattering losses. 
Mie scattering losses result from compositional fluctuations as well as structural inhomogeneities. Moreover, manufacturing defects also results in light scattering outside the fibre.

Waveguide scattering losses take place due to variations in the fibre's core diameter, core cladding interface weaknesses as well as due to changes in refractive indices of either core or cladding.

\section{Global trends as a result of industrial fibre-optic development}

Fibre-optic market has been seeing immense growth primarily due to market demand and technological advances as elaborated in the previous section. All-Optical networks and multi-terabit networks are two major trends. The former objectively aims to process all signals within the optical chains without ever converting to the electrical domain in anyway. Yet, the majority of signal routing, processing, and the network-based routing has occurred in the electrical domain where all the optical signals are converted. Once these electrical signals are process, routed and switched to their destination, the signals are reconverted to optical signals which are then transmitted to longer distances. This technique is commonly termed as the O-E-O process. This process acts as a performance bottleneck as it severely limits network data transfer and routing rates which gave way to research and development trends in the fibre-optic industry to eliminate electronics completely from optical data transfer.

\subsection{All-Optical networks}

The advantage of All-optical networks is their capability to perform the entire signal processing, routing and switching in the optical domain where there is no need to switch to the electrical domain to cater for a data rate increase (Subramaniam et al. 1996). Existing fibre optic transmitters and receivers are only assembled to address one data transfer rate which is still a limitation if the data rate is to be increased further thereby requiring a system replacement. However, this would not be mandatory in an all-optical network. The trend, however, still suffers from a number of serious drawbacks. For instance, reading optical signal headers, on-thefly optical signal switching with the header content and real-time wavelength switching are a few such limitations that are still being investigated to achieve an all-optical network (See Figure 3 ).

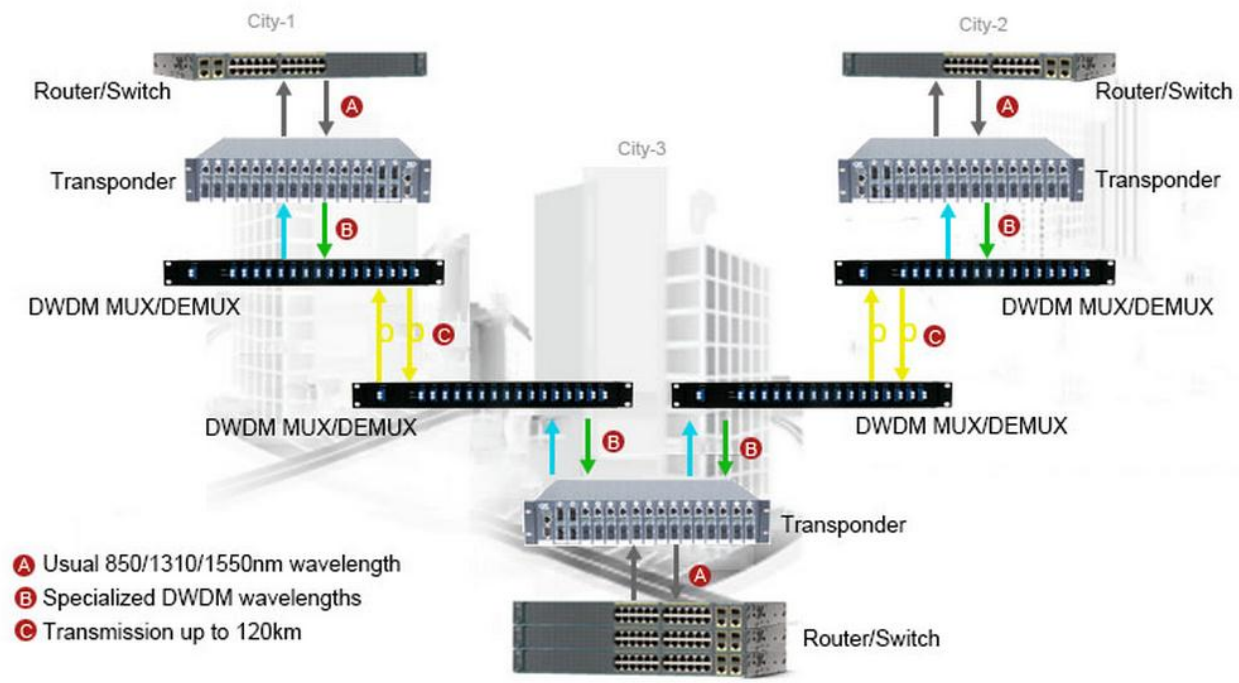

Figure 3: DWDM-based multi-terabit networks (Fibreopticshare, 2013)

\subsection{Multi-Terabit networks}

Dense wavelength division multiplexing (DWDM) is the optical signal multiplexing within the 1550 nanometre band in order to utilise the potential and cost of erbium doped fibre amplifiers (EDFAs). The EDFAs are capable of augmenting any optical signal within their operating range. The underlying DWDM opens the door for multi terabit transmission which is driven by an interest to obtain more bandwidth in fibre optic networks (Cvijetic 2012). A one terabit network is thus obtained by employing a 10Gb/s data rate in combination with 100 DWDM channels which can be extended to 40Gb/s at present via 100 DWDM channels. Research at present are focussing on higher bandwidth $100 \mathrm{~Gb} / \mathrm{s}$ systems which are very expensive and may only suit long-haul transmission systems. 


\subsection{Data supply and demand}

The fibre-optics industry at present is largely driven as a result of a worldwide shift to informationcentric communication. The trend has particularly taken hold since mid-1990s with the advent of the internet and the subsequent takeover of high-speed broadband market in the past 20 years. Till late 1990s, network resources were slow and expensive which lead to issues of sparse data densities and application domains that were restricted to single local area networks (LANs) which were catered by a limited number of CPUs and data processing units.

During the current decade, data clients started to improve as a result of advances in fibre optic communication due to research trends already presented in the previous section. Particularly, the move from analogue to digital transmission services resulted in a big increase in the data clients with data services sharing common networks expanding to wider network domains known as wide area networks (WANs).

As a result, digital communication networks increased exponentially in the previous decade with the rapid improvement in computational technologies and increase in distributed computation resources. For instance, the majority of network service providers in the West now provide video, internet and telephones services under a single fibre-optic medium with standard telecommunication equipments such as the television, computers and the telephone handsets. The data transfer medium for all these appliances have now started to blur as a result of high speed broadband for internet television applications and voice over IP (VOIP) protocols for internet telephony applications.

\subsection{Fibre-optic interface demand and internet accessibility}

At present, a large section of physical layer suppliers seem to originate from China. An increased access demand corroborates an increasing pressure on networks in the Far East where VSDL retrofits are replacing ASDL modems at a rapid pace. Consequently, internet access rate report South Korea at $52 \%$ where $91 \%$ of South Korean households use latest broadband technologies compared to only $20 \%$ in the United States (EDN, 2003).

\subsection{Bitrate convergence and market integration of high-speed fibre optics}

The current bit rate convergence is still predominantly using 10Gbps nodes. Therefore, chipmakers still focus on circuits and design rules aiming the 10Gbps market. These rules adhere to the synchronous optical network (SONET) jitter requirements. This characteristic facilitates the usage of low-cost CMOS processes as well as a large fabrication capacity. The protocol-agnostic transceiver chips, which can be used for Ethernet or fibre channel systems, take advantage of this reduced signalling rate at $10 \mathrm{Gbps}$ and offer variable deployment and operations models for a robust network operation. Consequently, instead of allocating fibres or wavelengths for specific traffic types, allocations can be made by the operators in real time which reduces operating costs and permits them to handle dynamic demand and mixed data traffic.

At present, the market trend substantially limits the deployment of 40Gbps nodes which will potentially delay the deployment of large scale OC-768 to at least 3 years. Moreover, previous-generation technologies continue to grow which include Gigabit Ethernet as well. This makes it possible for large scale to take advantage of 1,2 and 10Gbps signalling rates (Chen et al. 2006).

\subsection{Footprint tracking and multi-source-agreements (MSAs)}

Optical transceivers are highly in demand nowadays however their form-factor or footprint still remains are significant challenge (Jinyu et al. 2009). Small-form-factor-pluggables (SFPs) decouple optics from line cards. This capability makes it possible for the line card to cater several applications simply by replacing the module whereas with fixed optics devices, the assembly sticks to a single line card. With the change of application, the footprint of these MSAs changes as well. The 300-pin MSA acts as a default standard module footprint optimised for the OC-192 service. Any modules that comply with this 300-pin standard provide a system wide data path containing 16 lines operating at $622 \mathrm{MBps}$ mapped onto a 10Gbps optical feed channel. These transponders (XFP-MSAs) fall into four general categories (CISCO, 2013):

- Shortwave/Short-reach transponders: This is an $850 \mathrm{~nm}$, multi-mode optical interface that use LC connectors and support fibre distance of up to 300 feet. Their limitation makes them suitable for facilityspecific, short-range connectivity.

- Short-reach/intra-office XFP interface: The 1310nm single-mode optical interface use LC connectors and support fibre distances of up to $2 \mathrm{~km}$ making them suitable for office-specific connectivity.

- Intermediate-reach/short-haul transponders: These are $1550 \mathrm{~nm}$ interfaces with a range of up to $20 \mathrm{~km}$.

- Long-reach/long-haul transponders: These 1550nm long-haul single-model optical interfaces are capable of providing fibre-optic range of up to $80 \mathrm{~km}$. 
MSA applications are largely focussed on high polarisation mode dispersion (PMD) fibre applications where the maximum likelihood sequence estimation (MLSE) technology can monitor and resolve errors occurring due to time variant effects (Roudas et al. 2004). These networks present increasingly challenging issues that include chromatic dispersion (CD), PMD and other nonlinear disparities that optical compensation modules and amplifiers that in turn increase network complexity, induce noise and reduce optical signal-to-noise ratio (OSNR) and reduce network range.

Additionally, these interfaces provide support for low latency data centre applications such as the Server Time Protocol which is highly sensitive to latency introduced by CD optical fibre. At the enterprise level, assembly and installation costs of dispersion compensation units (DCU) and optical fibre can be eliminated. In low-end markets, this results in an overall $10 \%$ cost reduction in the overall installation costs. In ultra long-haul (ULH) applications improved CD and PMD is transformed into better performance whereas the nonlinear effects (NLE) impairment can be improved via additional optical signal-to-noise ration margins to further improve system performance.

\subsection{Vertical cavity surface emitting lasers (VCSELs)}

VCSELs are a type of semiconductor laser diode predominantly used in optical fibre data transmission, analogue broadband signal transmission, absorption spectroscopy, etc. The technology promises to revolutionise fibre optic communication by improving network efficiency and enhancing data transfer rates (Sompie and Uranus 2011). VCSELs (pronounced vixel) emit coherent energy which is perpendicular to the in-layer boundaries. Laser diodes in VCSELs exhibit boundaries as horizontal planes which results in the VCSEL outputs to emerge vertically. The VCSEL technology provided several benefits over edge emitting diodes. The former is more cost-effective to manufacture in larger quantities and is more efficient. Moreover, VCSELs require less current to generate a coherent energy output. Also, VCSELs bear a narrow and finely circular beam when compared to the traditional edge emitters which makes it feasible to draw energy from the device into the optical fibre. Another challenges faced by production engineering firms is to develop high power VCSEL devices that are capable of operating at an emission wavelength of $1550 \mathrm{~nm}$. These longer wavelength diodes are likely to extend device operation from short-haul and ultra-short-haul services to medium-reach devices and applications.

\subsection{Super PHYs}

Quality-of-service (QOS) levels are monitored in fibre optic systems via channels monitors. These monitors function at the transport and network layers while integrated with the routing and sequencing functions. The functions are defined via the OSI model's $3^{\text {rd }}$ layer and the flow-control and recovery functions at Layer 4. If the network nodes and physical layer has a clear channel, the underlying devices can easily detect and efficiently resolve the bit stream and the data link. Moreover, the network layer acts deterministically and prevents any system failures due to power stoppage or cooling system failures.

Devices operating at the physical layer of these fibre-optic systems can now detect channel variability with the QOS standing for the quality of signal. At this level, monitoring functions are able to collate and report channel condition diagnostic data to initiate routing decisions and trigger and guide maintenance requests and activities. As part of the channel optimisation schemes, these monitors are also used to minimise bit-error-rate statistics.

Physical layer (PHY) chips connect link layer devices to a physical medium which is normally fibre optic cable (Feng and Sandhu 2007). PHY chips incorporate servo-based data-eye optimisation. These adaptive technologies mainly focus on copper-based systems aimed at improving T3, Gigabit Ethernet IO, and backplane transceivers. At present, optical multi-source-agreements (MSAs) have not started exploiting the full potential of PHY chips' reporting capabilities. These capabilities are primarily meant to raise media system awareness and/or interface-specific situations. Recent extensions to small-form-factor pluggable (SFF/SFP) MSA definitions provide access to the operating parameters of an optical module. These parameters include system temperature, voltage, bias current, and signal strength. Limits and thresholds on these parameters can be used to monitor outlying values to distinguish system defects and/or poor operating and/or signal conditions.

\subsection{Micro Opto-electromechanical Systems (MOEMS)}

With the advent of very large scale integration (VLSI) technology, not only mechanical, but optical units such as beam splitters, lenses and reflectors can also be integrated in miniature semiconductor technologies which are now giving way to a new type of fibre-optics trend known as micro-optics (Ibrahim and Fayek 2006). The devices are well-proven against mechanical vibrations which lead to the next step of integrating the microoptical devices with the micromechanical devices to obtain micro-opto-electromechanical systems (MOEMS). 
MOEMS are 3D integrated optical circuits that comprise of self-calibrated and self-tested optical elements obtained via high-precision mechanical actuation. These systems have revolutionised the fibre-optics industry due to an outstanding growth in bandwidth capacity and networking capabilities of lightwave systems. MOEMS are low power consumption and low cost devices. According to Dragoman and Dragoman (2001), three trends and directions can be attributed to MOEMS-based systems (See Figure 4):

- Improvement of lasers, photo detectors, filters and mirrors in order to calibrate their characteristics in a wide range of embedded electronics and communication devices

- The assembly of MOEMS devices such as switches, attenuators and free-space setups including Michelson interferometers

- Development of sophisticated signal processing devices such as spectrometers, high-performance harddrives and optical computers

The technology is further extended to new frontiers via the nanotechnology to the nanometre scale with new engineering designs commonly known as the nano-electromechanical systems (NEMS). Both the MOEMS and NEMS are used in the fibre optics technology in the development of wired and wireless, electrical and mechanical sensors, free-space optical interconnections, WDMs and switches.

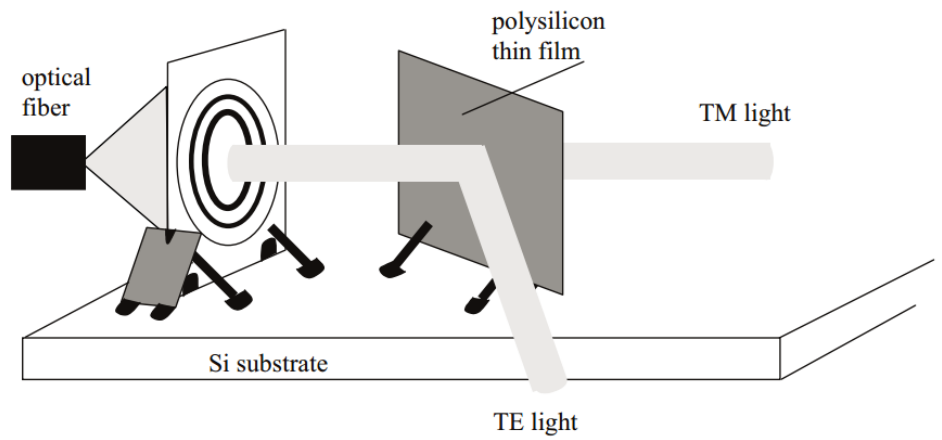

Figure 4: A fire-optic micro-machined beam splitter based on the polarisation principle (Dragoman and Dragoman 2001)

\section{Fibre-optics research driving the recent fibre-optic advancements}

The first commercial 40Gbps modem is reported by Roberts et al. (2009) which presented the evaluation of the CMOS ASIC technology integrating the required analogue to digital converters (ADC) and digital signal processing (DSP) units required to access and manipulate the opto-electrical signals. The low power requirement of these devices was estimated to be low-cost. It was anticipated that the optical coherent detection can facilitate optical transmission systems of up to 1 Tbps (terabytes per second) per wavelength. The block diagram of a DP-QPSK coherent transceiver with ADC blocks for analogue to digital conversion, an integrate finite impulse response (FIR) linear filter and a least mean square (LMS) algorithm to facilitate a 40Gbps data transfer rate (See Figure 5). A commercialised version of the same is shown in Figure 6.

In order to achieve transfer rates of up to $100 \mathrm{Gbps}$ at $50 \mathrm{Ghz}$ channel spacing can be achieved at two waves with each at $14 \mathrm{GBaud}$ or a single $26 \mathrm{GBaud}$. The selection of one of these may prove cost-effective compared to the other (Han et al. 2012).

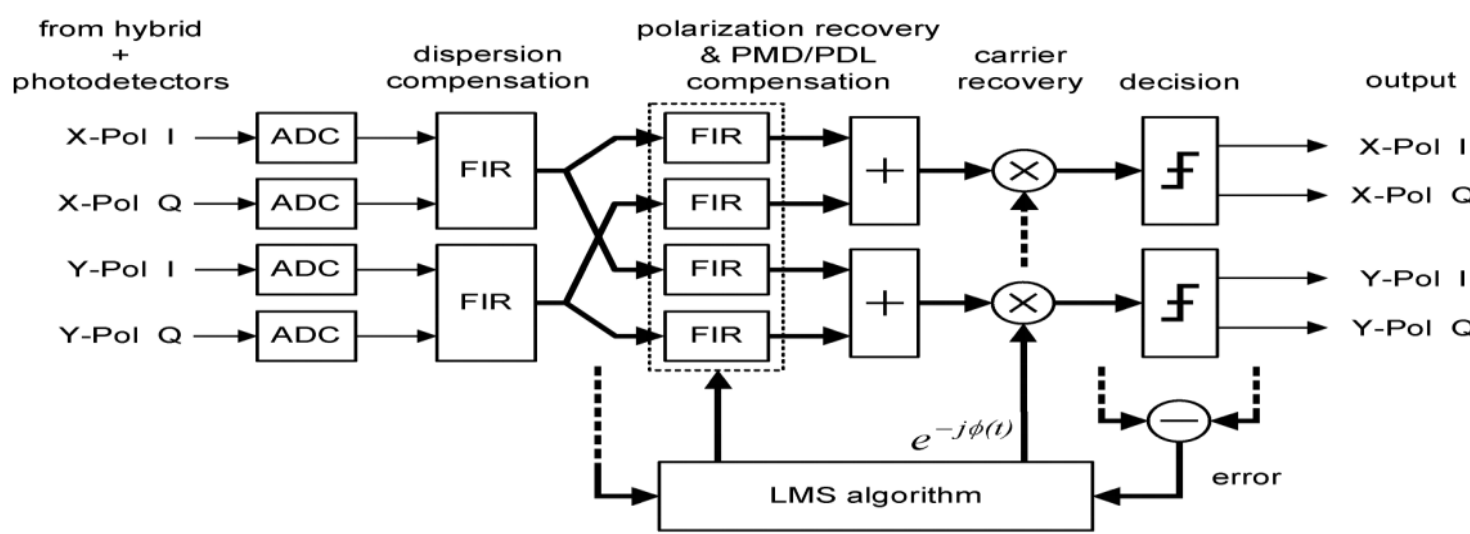

Figure 5: Block diagram of a 40Gbps DP-QPSK coherent transceiver as adapter (Laperle et al. 2008) 


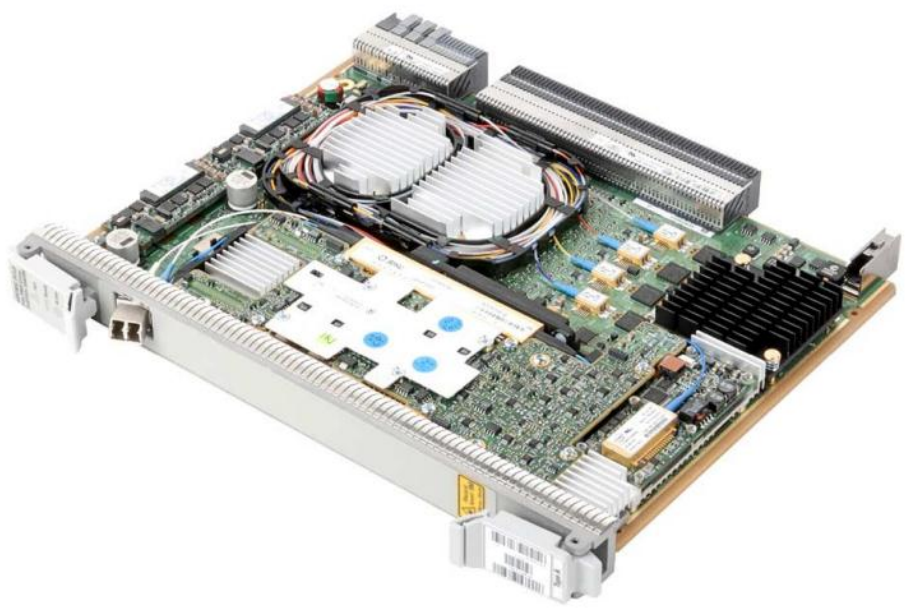

Figure 6: A commercial 40 Gbps DP-QPSK coherent transceiver (Roberts et al. 2009)

Lately, fibre optic research trend has focussed more on data transfer, architecture improvement, noise reduction, and QOS issues. Reljin et al. (2005) investigated the impact of radiation induced loss in fibre optic digital communication links and confirmed the validity of the proposed processing method via discrete data obtained by the sampling oscilloscope. Recent research multiplexing fibre optic sensing technology has revealed optical amplification methodologies and lasing multiplexing systems capable of handling robust, remote and distributed Brillouin networks (Perez-Herrera and Lopez-Amo 2013). Recent advances in optical communication cover optical modulators, optical switches including diffractive DMD switches and reconfigurable optical add-drop multiplexers. The review also presents substantial improvements in the domains of advanced coding and modulation schemes, OFDM, protection schemes and cross-layer design of optical networks. This also includes the design of impairment-aware routing and wavelength assignment and energy-efficient, cross-layer signal processing algorithms. The advances have further focused on network control and management protocols and implementations and dynamic service provisioning. Additional focus has also been on performance comparison of various cable types such as cat 5e copper cables and 4-core multimode optic fibre cables (Unger and Gough 2008). Focus has also been on alarm-condition detection and localisation via Rayleigh scattering for fibre optic bending sensors (Spirin et al. 2002) and improved optic sensor fabrication via micromachining (Rao and Ran 2013). The effects of PMD on channel capacity of coherent fibre optic systems were investigated by (Goebel et al. 2007). Moreover, work has also focussed on phase-sensitive parametric amplifiers (Tang et al. 2005) and dispersion maps (Fischer et al. 2006). Based on an increasingly focussed research trend on fibre optic network protocols, speed and quality optimisation techniques, it can be ascertained that the fibre optic communication domain is very likely to gain further improvement in near future.

\section{Critical Review and Conclusion}

There is no concrete trend prevalent in the fibre-optic-communication technology though the focus seems to be on the main area network (MAN) technologies covering the wavelengths of up to $1550 \mathrm{~nm}$. The underlying data traffic continues to grow at an overwhelming pace despite the unavailability of a homogenous traffic distribution model. This results in geographically converging dense traffic patterns. This phenomenon is further intensifying as the wireless LAN and public hotspot platforms increase in numbers. Due to the current economical climate, applications are still convergent on $10 \mathrm{Gbps}$ nodes. The so-called protocol agnostic technologies are thus helping platform developers, service providers as well as the general consumer. Despite a sluggish market acceptance of higher-speed nodes, vendors are improving fibre optic technologies including the VCSEL and MOEMS devices. These devices are now proving robust and presenting outstanding performances.

\section{References}

[1]. Agrawal, G.P. (1997) Fibre Optic Communication Systems 4 (ed.) Wiley : 1997

[2]. Charlet, G. (2008) Coherent detection associated with digital signal processing for fiber optics communication. Comptes Rendus Physique, 9(9-10), pp.1012-1030.

[3]. Chen, D.Z., Xia, T.J., Wellbrock, G., Mamyshev, P., Zik, J.J., Penticost, S., Grosso, G., Dismukes, A., Perrier, P. and Fevrier, H. (2006) New Field Trial Distance Record of $3040 \mathrm{~km}$ on Wide Reach WDM with 10 and 40 Gbps Transmission Including OC-768 Traffic Without Regeneration. Optical Fiber Communication Conference, 2006 and the 2006 National Fiber Optic Engineers Conference. OFC 2006, pp.1-3. Available from.

[4]. Cheng, W.-M. and Tsao, S.-L. (2005) Performance enhancement on mechanical and electrical effects for on-line fiber-optic bending loss measurement. Optik - International Journal for Light and Electron Optics, 116(5), pp.201-210. 
[5]. CISCO (2013) Extended Performance 10 Gbps Full-Band Tunable Transponder Card [Online] Available at http://www.cisco.com/c/en/us/products/collateral/optical-networking/ons-15454-series-multiservice-provisioning-

platforms/data sheet c78-580763.html [Accessed: 14th March, 2014]

[6]. Cvijetic, M. (2012) Modulation and coding techniques, and optical networking technologies enabling multi terabit bandwidth delivery. Lasers and Electro-Optics (CLEO), 2012 Conference on, pp.1-2. Available from.

[7]. Dragoman, D. and Dragoman, M. (2001) Micro/nano-optoeletromechanical systems. Progress in Quantum Electronics, 25(5-6), pp.229-290.

[8]. Feng, X. and Sandhu, S. (2007) PHY-layer network coding for broadcast channel with side information. Information Theory Workshop, 2007. ITW'07. IEEE, pp.108-113. Available from.

[9]. Fiberopticshare (2013) Trends in Fibre Optic Communication Industry [online] Article available at < http://www.fiberopticshare.com/trends-in-fiber-optic-communication-industry.html > [Accessed: $14^{\text {th }}$ March, 2014]

[10]. Fischer, J.K., Bunge, C.A., Jamshidi, K., Louchet, H. and Petermann, K. (2006) Equivalent dispersion maps in fibre-optic communication systems. Optical Communications, 2006. ECOC 2006. European Conference on, pp.1-2. Available from.

[11]. Goebel, B., Kuschnerov, M. and Hanik, N. (2007) On the Effect of Polarisation-Mode Dispersion on the Channel Capacity of Coherent Fibre-optic Communication Systems. Transparent Optical Networks, 2007. ICTON '07. 9th International Conference on, 1, pp.155-159. Available from.

[12]. Han, S., Kuang-Tsan, W., Mcnicol, J., Mitchell, M., Dangui, V., Vanleeuwen, M., Rahn, J., Grubb, S., Nagarajan, R., Ziari, M., Corzine, S., Evans, P., Kato, M., Kish, F. and Welch, D. (2012) Recent advancements in fiber optic transmission. Wireless and Optical Communications Conference (WOCC), 2012 21st Annual, pp.14-19. Available from.

[13]. Ibrahim, H. and Fayek, R. (2006) Adaptive MOEMS for Dynamic Beam Focusing. Optoelectronic and Microelectronic Materials and Devices, 2006 Conference on, pp.216-219. Available from.

[14]. Israelshon, J. (2003) Seven Trends in Fibre Optic Communication, [Online] Available at < http://edn.com/electronicsblogs/other/4331731/Seven-trends-in-fiber-optic-communication > [Accessed: 14th March, 2014]

[15]. Jinyu, M., Griffin, R., Goodall, T. and Zheng, H. (2009) Advanced InP technology for high performance 40 Gb/s (RZ-) DQPSK transponder. Communications and Photonics Conference and Exhibition (ACP), 2009 Asia, 2009-Supplement, pp.1-6. Available from.

[16]. Laperle, C., Villeneuve, B., Zhuhong, Z., Mcghan, D., Han, S. and O'sullivan, M. (2008) WDM Performance and PMD Tolerance of a Coherent 40-Gbit/s Dual-Polarization QPSK Transceiver. Lightwave Technology, Journal of, 26(1), pp.168-175.

[17]. Lines, M.E. (1984) Scattering losses in optic fiber materials. II. Numerical estimates. Journal of Applied Physics, 55(11), pp.40584063.

[18]. Makouei, S. (2013) Characteristics comparison of the ZDSFs based on the triangular graded index and step index single-mode optical fibers. Optics Communications, 291(0), pp.202-206.

[19]. Pedrola, O., Rumley, S., Careglio, D., Klinkowski, M., Gaumier, C. and Solé-Pareta, J. (2011) Performance overview of the quasisynchronous operation mode in optical burst switching (OBS) networks. Optical Switching and Networking, 8(1), pp.32-45.

[20]. Perez-Herrera, R.A. and Lopez-Amo, M. (2013) Fiber optic sensor networks. Optical Fiber Technology, 19(6, Part B), pp.689-699.

[21]. Rao, Y.-J. and Ran, Z.-L. (2013) Optic fiber sensors fabricated by laser-micromachining. Optical Fiber Technology, 19(6, Part B), pp.808-821.

[22]. Reljin, I., Reljin, B., Sreckovic, M., Marinovic, A., Kovacevic, M. and Tomic, Z. (2005) A way to determine radiation-induced loss in fiber-optic digital communication link. Optical Fiber Technology, 11(3), pp.286-291.

[23]. Roberts, K., O'sullivan, M., Kuang-Tsan, W., Han, S., Awadalla, A., Krause, D. J. and Laperle, C. (2009) Performance of DualPolarization QPSK for Optical Transport Systems. Lightwave Technology, Journal of, 27(16), pp.3546-3559.

[24]. Roudas, I., Piech, G.A., Mlejnek, M., Mauro, Y., Chowdhury, D.Q. and Vasilyev, M. (2004) Coherent frequency-selective polarimeter for polarization-mode dispersion monitoring. Lightwave Technology, Journal of, 22(4), pp.953-967.

[25]. Routray, S.K. (2014) The Changing Trends of Optical Communication. Potentials, IEEE, 33(1), pp.28-33.

[26]. Sompie, S.R. and Uranus, H.P. (2011) Design of single-transversal-mode VCSEL with photonic crystal holey cladding. Electrical Engineering and Informatics (ICEEI), 2011 International Conference on, pp.1-5. Available from. ISSN $2155-6822$.

[27]. Spirin, V.V., Shlyagin, M.G., Miridonov, S.V. and Swart, P.L. (2002) Alarm-condition detection and localization using Rayleigh scattering for a fiber-optic bending sensor with an unmodulated light source. Optics Communications, 205(1-3), pp.37-41.

[28]. Subramaniam, S.S., Azizoglu, M. and Somani, A.K. (1996) All-optical networks with sparse wavelength conversion. Networking, IEEE/ACM Transactions on, 4(4), pp.544-557.

[29]. Tang, R., Devgan, P., Grigoryan, V.S. and Kumar, P. (2005) Inline frequency-non-degenerate phase-sensitive fibre parametric amplifier for fibre-optic communication. Electronics Letters, 41(19), pp.1072-1074.

[30]. Unger, N. and Gough, O. (2008) Life cycle considerations about optic fibre cable and copper cable systems: a case study. Journal of Cleaner Production, 16(14), pp.1517-1525.

[31]. Xu, Y., Jones, N.B., Fothergill, J.C. and Hanning, C.D. (2001) Error analysis of two-wavelength absorption-based fibre-optic sensors. Optics and Lasers in Engineering, 36(6), pp.607-615.

[32]. Yaman, F., Qiang, L., Radic, S. and Agrawal, G.P. (2006) Fiber-optic parametric amplifiers in the presence of polarization-mode dispersion and polarization-dependent loss. Lightwave Technology, Journal of, 24(8), pp.3088-3096. 